Published Version

Originally published at:

Kiening, Christian (2002). Historische Anthropologie in literaturwissenschaftlicher Perspektive. Historische Anthropologie, 10:305-307. 


\title{
Historische Anthropologie in literaturwissenschaftlicher Perspektive
}

\author{
von Christian Kiening
}

Historische Anthropologie scheint in
den letzten Jahren zu einem Forschungs-
feld geworden zu sein, in dem sich Inter-
essen verschiedener Disziplinen in exem-
plarischer Weise begegnen und kreuzen
Entwickelte zum Beispiel die Geschichts-
wissenschaft eine erhöhte Aufmerksam-
keit für anthropologische Dispositionen
menschliche Elementarerfahrungen und
mikrohistorische Beobachtungseinheiten so richtete die Literaturwissenschaft das Augenmerk auf Texte als Produkte gesellschaftlicher Kommunikation, Repräsentationen menschlichen Handelns und Konstruktionen kulturellen Sinns. Besonders eng scheint die Berührung im Bereich mittelalterlicher Literatur und Kultur: Hier existiert weder ein ausdifferenziertes System der Anthropologie noch eines der Literatur; die wissenschaftliche Beschreibung muß deshalb versuchen, Muster und Strukturen herausarbeitend kulturelle Elemente in ihren jeweiligen Handlungszusammenhängen und pragmatisch fundierten Sinngefügen zu erfassen - wie ähnlich die Ethnologie fremde kulturelle Formationen. Jeweils zielt der anthropologische Zugang weniger auf eine Reduktion als auf eine Entfaltung kultureller Vielfalt: Er bringt Widerstimmiges und scheinbar Marginales ans Licht und wirkt auf diese Weise einer homogenisierenden Vereinnahmung des zeitlich oder räumlich Fremden entgegen.

Indes ist die Begegnung der Fächer nicht ohne Gegenläufigkeiten. Zwar ist in der Geschichtswissenschaft die Sensibilität für die sprachliche und textliche, die materielle und semiotische Dimension von Überlie- ferung gestiegen, doch der, linguistic turn trägt wie zahlreiche andere ,Wenden', die einander in jüngster Zeit inflationär überbieten, weit mehr programmatische als faktische Züge. Geschichtswissenschaftliche Arbeiten zur Historischen Anthropologie halten nicht selten an einem Begriff der Quelle fest, der diese als vielleicht perspektivisch verzerrte, im ganzen aber mehr oder weniger unmittelbare Wiedergabe von Wirklichkeit versteht. Auch suggerieren sie, allein die Konzentration auf bestimmte Themenfelder (Geschlecht, Familie/Verwandtschaft, Geburt, Tod, Körper, Sexualität, Ernährung, Raum, Zeit, Fremdheit etc.) erlaube es bereits, dem jeweils historisch Spezifischen menschliche Elementarerfahrungen abzulesen.

Es liegt auf der Hand, daß komplexere Formen der Überlieferung komplexere Entwürfe anthropologischer Dispositionen bieten und dementsprechend komplexere Methoden der Analyse benötigen. Literarische Texte sind modellbildende Systeme zweiter Ordnung. Sie betreiben nicht nur höheren Aufwand in der Formung der Sprache und der Entwicklung des Imaginären. Sie verknüpfen auch, indem sie sich seligierend und kombinierend auf vorhandene Gattungen und Diskurse beziehen, den Entwurf anthropologischer Dimensionen mit einer Ausstellung oder sogar expliziten Thematisierung der jeweils eigenen Modalitäten der Sinnkonstitution. Doch geht es nicht nur um unterschiedliche Komplexitäten: Die literaturwissenschaftliche Perspektive wird auch dort, wo pragmatische Texttypen zur Diskussion stehen, den Bedin- 
gungen der Literarisierung gelten und diese weniger als brechende Linsen begreifen, die die Wahrnehmung des Realen verhindern, denn als mediale Voraussetzungen, die die Möglichkeit, bestimmte (etwa anthropologische) Sachverhalte zu artikulieren, (mit)determinieren. Unterschiedliche Erkenntnisinteressen bedingen unterschiedliche Fokussierungen. Der Literaturwissenschaftler richtet die Aufmerksamkeit in stärkerem $\mathrm{Maße}$ auf die historischen $\mathrm{Ob}$ jekte in ihrer materiellen und semiotischen Dimension, der Historiker auf die jeweiligen Aussageformen in ihrer Verflechtung mit Ereignissen, Mentalitätten und Strukturen.

Die unterschiedlichen Interessen können sich ergänzen, sie können aber auch, und dies selbst dann, wenn sie die gleichen Objekte tangieren, im bloßen Nebeneinander verbleiben. Das zu verhindern, mag es sinnvoll sein, statt von inhaltlichen oder thematischen eher von methodischen und systematischen Aspekten auszugehen. Gemeinsame Ansatzpunkte lassen sich zumindest in vierfacher Hinsicht formulieren:

(1) In der Bezugnahme auf Ansätze der Kultur- und Sozialanthropologie. Diese hat Methoden ausgebildet, den Zusammenhang von Einzelelementen im (wie auch immer fragmentarischen) Ganzen einer kulturellen Formation zu erfassen, und hat sich mit der Frage beschäftigt, wie fremde Sinnsysteme im Spannungsfeld von Beobachter und Beobachtetem zu repräsentieren sind. Mediävistisch relevant ist das eine, wenn Überlagerungen zwischen sozialen Feldern beschrieben werden sollen, die nur ansatzweise Institutionalisierungen und funktionale Ausdifferenzierungen kennen, das andere, wenn neben Kontinuitäten auch Diskontinuitäten zwischen mittelalterlichen und neuzeitlichen Sinnsystemen in den Blick treten sollen.

(2) In der Fokussierung der Dialektik von Mikro- und Makrogeschichte. Haben sich historische Untersuchungen auf überschaubare Gemeinschaften und zeitlich oder räumlich kohärente Kulturkomplexe konzentriert, an denen die Interaktion sozialer Praktiken exemplarisch zu studieren ist, so haben literaturwissenschaftliche Untersuchungen einzelne Überlieferungsträger und -zusammenhänge in den Mittelpunkt gestellt, aus denen mediale, pragmatische und diskursive Existenzbedingungen von Sinngefügen besonders deutlich hervorgehen können. In jedem Fall gilt es, mikrohistorische Perspektiven auf kleine Einheiten mit makrohistorischen Perspektiven zu verknüpfen, die modellhaft in Längsschnitten Stabilitäten und Umbrüche kultureller Formationen sichtbar machen.

(3) In der Entwicklung von Formen selbstreflexiver Modellbildung. Begreift man den Gegenstand als einen, der in seiner historischen Eigenlogik dem Gegenwartshorizont der Forschung nicht ohne weiteres inserierbar ist, ergibt sich die Notwendigkeit, mit analytischen Modellen zu operieren, die einerseits modifizierbar sein müssen, andererseits den je eigenen Fokus bzw. das je eigene Konstruktionsprinzip erkennen lassen. Es geht hier also nicht nur, wie manchmal suggeriert, um die unvermeidliche Subjektivität und Standpunktgebundenheit der Analyse, sondern um das kontrollierte, einer Präzisierung des Beschreibungsinventars dienende Experiment mit wechselnden kleineren und größeren Distanzen - das Experiment einer Hermeneutik, die prozessualen und infiniten Charakter hat nicht nur aufgrund der Unabgeschlossenheit historischer Erklärungsmöglichkeiten, sondern auch aufgrund der prinzipiellen Nicht-Verfügbarkeit des Gegenstands.

(4) In der Profilierung von Verfahrensweisen einer Historischen Semantik. Die von Historikern wie Literaturwissenschaftlern bemühte Metapher der ,thick description \%,dichten Beschreibung' (Geertz) kann, so mit ihr eine besondere Aufmerksamkeit für historisch-eigenkulturelle Logiken gemeint ist, im Sinne einer Historischen Semantik konkretisiert werden. Im Zentrum stünde dann die Frage nach den 
Bedingungen, die $\mathrm{zu}$ einem bestimmten Zeitpunkt und in einer bestimmten Situation Bedeutung ermöglichen, regulieren und stabilisieren: sprachliche oder bildliche, epistemische oder ontologische, anthropologische oder psychologische Bedingungen, die wiederum historischem Wandel unterworfen sind. Historische Semantik richtet den Blick darauf, daß Bedeutung prinzipiell unfest ist, ihre Fluktuation aber auch immer wieder (und über längere Zeit) eingeschränkt oder stillgestellt wird. Sie fragt nach dem, was historischen Gesellschaften und Individuen an Möglichkeiten der Sinnbildung zur Verfügung steht. Sie durchleuchtet die Arten und Wei- sen, in denen Wissen durchgesetzt, aber auch in Frage gestellt, Erinnerung institutionell gesichert, aber auch transformiert wird. Sie erforscht Diskurse und Institutionen, Praktiken, Regeln und Strategien, die gesellschaftliche Kommunikation und ästhetische Imagination bestimmen.

Diese nur ausschnittweise skizzierten Aspekte machen deutlich, wie wenig sich die hier geschichts-, dort literaturwissenschaftliche Annäherung an Phänomene des Anthropologischen schon auf eine gemeinsame Basis stützen kann, wie viel sie aber auch an Erkenntnis- und Forschungspotentialen im Bereich symbolischer Ordnungen noch wird entdecken können. ${ }^{1}$

1 Vgl. Christian Kiening, Anthropologische Zugänge zur mittelalterlichen Literatur. Konzepte - Ansätze - Perspektiven, in: Forschungsberichte zur Germanistischen Mediävistik 5/1, hg. von Hans-Jochen Schiewer, Bern u. a. 1996, 11-129; Ders., Zwischen Körper und Schrift. Texte vor dem Zeitalter der Literatur [erscheint 2002]. 